\title{
PLURIHARMONIC MORPHISMS
}

\author{
E. LOUBEAU
}

\begin{abstract}
Pluriharmonic maps form an important class of harmonic maps which includes \pm holomorphic maps. We study their morphisms, in particular the inter-relationships between $(1,1)$-geodesic, pluriharmonic and tholomorphic maps. Then we characterise pluriharmonic morphisms between complex manifolds as tholomorphic maps. We make a special study of the situation where the domain is Hermitian and the target is Kähler, pluriharmonic morphisms having an extra property.
\end{abstract}

\section{Introduction}

One of the most important properties of harmonic maps is that, when mapping from a two-dimensional domain, the energy functional is invariant under conformal changes of the metric on that domain. We can therefore talk of harmonic maps from Riemann surfaces and their study has been prolific (cf. [2,3]). As any Hermitian structure on a Riemann surface is Kähler, harmonic maps from a Riemann surface are harmonic with respect to any Kähler metric on the domain. Maps which generalise this property to higher dimensions are called pluriharmonic maps. Harmonic morphisms between Riemannian manifolds are maps which pull back germs of harmonic functions to germs of harmonic functions. They were characterised independently by B. Fuglede in [4] and T. Ishihara in [6] as horizontally weakly conformal harmonic maps. A consequence of this result is that harmonic morphisms pull back harmonic maps to harmonic maps as well.

Our aim is to generalise the idea of harmonic morphism to maps which pull back pluriharmonic functions to pluriharmonic functions; we shall call such mappings pluriharmonic morphisms. First, we recall some types of Hermitian structures, concentrating on the geometrical interpretations of their definitions. These results enable us to compare how and when the notions of $(1,1)$-geodesic, pluriharmonicity and holomorphicity overlap. In particular, we show that, for maps between Hermitian manifolds, the class of \pm holomorphic maps is included in the class of pluriharmonic maps pre-

Received November 15, 1996. 
cisely when the complex structure carried by the target manifold is Kähler. We find that a necessary and sufficient condition for a map between complex manifolds to be a pluriharmonic morphism is that it be a \pm holomorphic map. Combining this characterisation of pluriharmonic morphisms with an earlier result, we observe that pluriharmonic morphisms from Hermitian to Kähler manifolds will pull back pluriharmonic maps to pluriharmonic maps.

I would like to thank J. C. Wood for painstakingly supervising this work and I. Calinov and R. Pantilie for pointing out some mistakes.

\section{Almost Hermitian manifolds.}

Recall that an almost Hermitian manifold $(M, J, g)$ is an even dimensional manifold $M^{2 n}$ equipped with an almost complex structure $J$ and a Hermitian metric $g$. On such a manifold we consider an adapted unitary frame $\left\{e_{1}, J e_{1}, \ldots, e_{n}, J e_{n}\right\}$. The complexified tangent space $T^{\mathrm{C}} M$ splits into $T^{(1,0)} M$ and $T^{(0,1)} M$ which are the eigenspaces of the endomorphism $J$ with eigenvalues $i$ and $-i$, respectively. We define $\left\{\theta_{j}=\frac{1}{\sqrt{2}}\left(e_{j}-i J e_{j}\right)\right\}_{j=1, \ldots, n}$ to be a unitary frame for $T^{(1,0)} M$ and $\left\{\theta_{\bar{J}}=\frac{1}{\sqrt{2}}\left(e_{j}+i J_{j}\right)\right\}_{j=1, \ldots, n}$ to be a unitary frame for $T^{(0,1)} M$. Let $\left\{\Theta^{i}, \Theta^{\bar{\imath}}\right\}_{i=1, \ldots, n}$ denotes the dual frame of $\left\{\theta_{i}, \theta_{\bar{\imath}}\right\}$, $\left\{\Theta^{i}\right\}_{i=1, \ldots, n}\left(\right.$ resp. $\left\{\Theta^{\bar{\imath}}\right\}_{i=1, \ldots, n}$ ) spans $T^{(1,0) *} M$ (resp. $T^{(0,1) *} M$ ). We shall denote by $g_{\bar{\jmath}}$ the quantity $g\left(\theta_{i}, \theta_{\bar{\jmath}}\right)$.

The fundamental 2 -form $\omega$ is defined as:

$$
\omega=\frac{i}{2 \pi} g_{\lambda \bar{\mu}} \Theta^{\lambda} \wedge \Theta^{\bar{\mu}}
$$

Definition 1. An almost Hermitian manifold $(M, J, g)$ is said to be:

1. $(1,2)$-symplectic if $(d \omega)^{1,2}=0$, i.e. $d \omega(\alpha, \beta, \bar{\gamma})=0$ for all $\alpha, \beta, \gamma \in$ $T^{(1,0)} M$.

2. Kähler if $\omega$ is closed, i.e. $d \omega=0$ and the almost complex structure is integrable.

3. cosymplectic if $d^{*} \omega=0$, where $d^{*}$ is the formal adjoint of $d$.

REMARK 1. The names quasi-Kähler or ${ }^{*} 0$ and semi-Kähler or almost semiKähler are sometimes used for (1,2)-symplectic and cosymplectic, respectively (cf. [10]). A list of sixteen different structures possible on an almost complex manifold as well as their classification can be found in Gray and Hervella [5].

$(1,2)$-Symplectic and Kähler manifolds can also be characterised in a more geometrical way:

If we denote by $\nabla$ the Levi-Civita connection then an almost Hermitian manifold $(M, J, g)$ is $(1,2)$-symplectic if and only if

$$
\nabla_{X} T^{(1,0)} M \subseteq T^{(1,0)} M \quad \forall X \in T^{(0,1)} M,
$$


whilst it is Kähler if and only if

$$
\nabla_{X} T^{(1,0)} M \subseteq T^{(1,0)} M \quad \forall X \in T M .
$$

Figure 1 on page 3 (based on that in [10]) shows how those different structures intersect one another, in particular that an integrable $(1,2)$-symplectic manifold is Kähler.

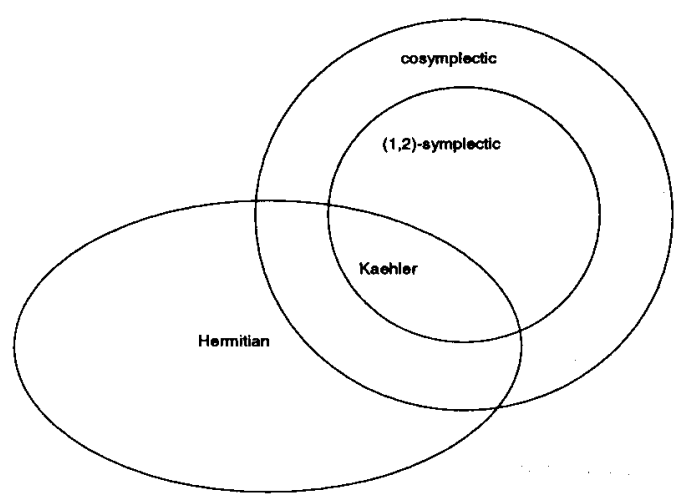

Figure 1. Intersection of the structures

The following standard proposition interprets the above definitions in terms of the metric $g$ and the Christoffel symbols $\Gamma_{J K}^{I}$ with respect to a unitary frame.

Proposition 1. [8] An almost Hermitian manifold $(M, J, g)$ is

1. integrable if and only if $\Gamma_{\lambda \mu}^{\bar{\alpha}}=0 \quad \forall \alpha, \lambda, \mu=1, \ldots, n$.

2. (1,2)-symplectic if and only if $\Gamma_{\bar{\lambda} \mu}^{\bar{\alpha}}=0 \quad \forall \alpha, \lambda$, $\mu$.

3. Kähler if and only if $J$ is integrable (equiv. $\Gamma_{\lambda \mu}^{\bar{\alpha}}=0 \quad \forall \alpha, \lambda, \mu$ ), and $(1,2)$ symplectic (equiv. $\left.\Gamma_{\bar{\mu} \lambda}^{\bar{\alpha}}=0 \quad \forall \alpha, \lambda, \mu\right)$ i.e. if and only if the only possibly nonzero Christoffel symbols are $\Gamma_{\mu \lambda}^{\alpha}$ and $\Gamma_{\bar{\mu}}^{\bar{\alpha}} \bar{\lambda}$.

4. cosymplectic if and only if $g^{\bar{\mu} \lambda} \Gamma_{\bar{\mu} \lambda}^{\alpha}=0$ for all $\alpha \in\{1, \ldots, n\}$.

\section{Pluriharmonic Morphisms.}

Let $N^{n}$ be an almost Hermitian manifold with a local adapted frame $\left(\vartheta_{1}, \vartheta_{\overline{1}}, \ldots, \vartheta_{n}, \vartheta_{\bar{n}}\right)$ and $X^{r}$ a Riemannian manifold with local coordinates $\left(\varpi_{1}, \ldots, \varpi_{r}\right)$. We denote by $\nabla$ the Riemannian connection on $X$ and by ${ }^{N} \Gamma_{A B}^{C}$ and ${ }^{X} \Gamma_{A B}^{C}$ the Christoffel symbols on $N$ and $X$

Definition 2. A map $\phi$ from an almost Hermitian manifold $N^{n}$ to a 
Riemannian manifold $X^{r}$ is called $(1,1)$-geodesic (see [3]) if the (1,1)-part of the second fundamental form of $\phi,\left({ }^{\phi} \nabla d \phi\right)^{(1,1)}$, is zero, i.e.:

$$
{ }^{\phi} \nabla d \phi(Z, \bar{W})=0 \quad \forall Z, W \in T^{(1,0)} N,
$$

where ${ }^{\phi} \nabla$ is the pull-back connection on $T^{*} M \otimes \phi^{-1} T X$.

Using the frame adapted to the almost complex structure on $T N$, Equation (2) can be written:

$$
\begin{aligned}
& \partial_{i \bar{\jmath}} \phi^{A}-{ }^{N} \Gamma_{i \bar{\jmath}}^{C} \partial_{C} \phi^{A}+{ }^{X} \Gamma_{J L}^{A} \partial_{i} \phi^{J} \partial_{\bar{\jmath}} \phi^{L}=0 \\
& \forall A \in\{1, \ldots, r\} \text { and } \forall i, j \in\{1, \ldots, n\},
\end{aligned}
$$

where we use the notations

$$
\partial_{C} \phi^{A} \text { for } d \phi^{A}\left(\vartheta_{C}\right) \text { and } \partial_{\bar{i} \bar{\jmath}} \phi A \text { for } d\left(d \phi^{A}\left(\vartheta_{i}\right)\right)\left(\vartheta_{\bar{\jmath}}\right) .
$$

If we suppose $N$ to be a Hermitian manifold with local complex coordinates $\left\{y^{1}, y^{\overline{1}}, \ldots, y^{n}, y^{\bar{n}}\right\}$ then locally $\phi$ satisfies:

$$
\begin{aligned}
& \frac{\partial^{2} \phi^{A}}{\partial y^{i} \partial y^{\bar{\jmath}}}-{ }^{N} \Gamma_{\bar{\imath}}^{C} \frac{\partial \phi^{A}}{\partial y^{C}}+{ }^{X} \Gamma_{J L}^{A} \frac{\partial \phi^{J}}{\partial y^{i}} \frac{\partial \phi^{L}}{\partial y^{\bar{\jmath}}}=0 \\
& \forall A \in\{1, \ldots, r\} \text { and } \forall i, j \in\{1, \ldots, n\} .
\end{aligned}
$$

Proposition 2. [3] $A(1,1)$-geodesic map from an almost Hermitian manifold to a Riemannian manifold is harmonic.

Definition 3. A smooth map from a complex manifold $N^{n}$ to a Riemannian manifold $X^{r}$ satisfying:

$$
\nabla^{(0,1)} d^{\prime} \phi=0
$$

is called a pluriharmonic map ([12]), where

$$
d^{\prime} \phi=\left.d \phi\right|_{T^{(1,0)} N^{n}},
$$

and

$$
\left(\nabla^{(0,1)} d^{\prime} \phi\right)(\bar{Z}, W)={ }^{\phi} \nabla_{\bar{Z}}\left(d^{\prime} \phi(W)\right)-d^{\prime} \phi\left(\bar{\partial}_{\bar{Z}} W\right) \quad \forall Z, W \in T^{(1,0)} N^{n} .
$$

A map is a pluriharmonic map if and only if its restriction to any holomorphic curve is harmonic (see [9, Prop. 1.1]).

We remark that:

$$
\nabla^{(0,1)} d^{\prime} \phi=0 \Leftrightarrow \nabla^{(1,0)} d^{\prime \prime} \phi=0 .
$$


The expression for the left-hand side of (4) in a local frame is:

$$
\begin{aligned}
& \left(\nabla^{(0,1)} d^{\prime} \phi\right)\left(\vartheta_{\bar{\jmath}}, \vartheta_{i}\right)= \\
& \left(\partial_{i \bar{\jmath}} \phi^{A}+{ }^{X} \Gamma_{J L}^{A} \partial_{i} \phi^{J} \partial_{\bar{\jmath}} \phi^{L}\right) \varpi_{A} .
\end{aligned}
$$

Hence the equation for pluriharmonicity of a map is

$$
\begin{aligned}
& \partial_{i \bar{\jmath}} \phi^{A}+{ }^{X} \Gamma_{J L}^{A} \partial_{i} \phi^{J} \partial_{\bar{\jmath}} \phi^{L}=0 \\
& \forall A \in\{1, \ldots, r\} \text { and } \forall i, j \in\{1, \ldots, n\} .
\end{aligned}
$$

REMARK 2. In local frames, the difference between pluriharmonic maps and $(1,1)$-geodesic maps is the extra terms of the type ${ }^{N} \Gamma_{i \bar{j}}^{C} \partial_{C} \phi^{I}$, which appear for $(1,1)$-geodesic maps.

If we suppose $N^{n}$ to be a Kähler manifold, then by Proposition 1.3, those terms disappear and the notions of $(1,1)$-geodesic maps and pluriharmonic maps coincide.

A pluriharmonic map on a complex manifold $N$ is harmonic with respect to any Kähler metric on $N$ and within this viewpoint can be seen as an extension of the notion of harmonic maps on surfaces (see Udagawa $[12,13]$ ).

Using Proposition 1 and the expressions of $(1,1)$-geodesic, pluriharmonic and \pm holomorphic maps in adapted frames, we study when those three different concepts coincide.

Definition 4. By local we shall mean defined on an open subset.

Proposition 3. Let $N^{n}$ and $X^{r}$ be Hermitian manifolds, then the condition that all (local) \pm holomorphic maps from $N^{n}$ to $X^{r}$ be pluriharmonic is equivalent to $X^{r}$ being Kähler. In particular, \pm holomorphic functions on a Hermitian manifold are always pluriharmonic.

Proof. The equation for a pluriharmonic map is:

$$
\begin{aligned}
& \frac{\partial^{2} \phi^{A}}{\partial y^{i} \partial y^{\bar{\jmath}}}+{ }^{X} \Gamma_{J L}^{A} \frac{\partial \phi^{J}}{\partial y^{i}} \frac{\partial \phi^{L}}{\partial y^{\bar{\jmath}}}=0 \\
& \forall A \in\{1, \overline{1}, \ldots, r, \bar{r}\} \text { and } \forall i, j \in\{1, \ldots, n\} .
\end{aligned}
$$

For a holomorphic map this reduces to the following equation:

$$
{ }^{X} \Gamma_{m \bar{p}}^{A} \frac{\partial \phi^{m}}{\partial y^{i}} \frac{\partial \phi^{\bar{p}}}{\partial y^{\bar{\jmath}}}=0 \quad \forall A \in\{1, \overline{1}, \ldots, r, \bar{r}\} \text { and } \forall i, j \in\{1, \ldots, n\},
$$

By choosing holomorphic maps with prescribed first derivatives at a point, Equation (5) shows that ${ }^{X} \Gamma_{m \bar{p}}^{A}=0 \quad \forall A, m, p$, so that, by Proposition $1, X$ is $(1,2)$-symplectic and so Kähler. 
The converse statement comes directly from the definitions.

Now we compare $(1,1)$-geodesic and \pm holomorphic.

Proposition 4. Let $N^{n}$ and $X^{r}$ be Hermitian manifolds, then the condition that all (local) \pm holomorphic maps from $N$ to $X$ be $(1,1)$-geodesic is equivalent to the condition that $N^{n}$ and $X^{r}$ be Kähler.

Proof. We equip $N^{n}$ with the local coordinates $\left(y^{1}, y^{\overline{1}}, \ldots, y^{n}, y^{\bar{n}}\right)$.

Let $\phi: N^{n} \rightarrow X^{r}$ be a holomorphic map, i.e.

$$
\frac{\partial \phi^{i}}{\partial y^{\bar{j}}}=0 \quad \forall i=1, \ldots, r, \forall j=1, \ldots, n .
$$

We recall that $\phi$ is a $(1,1)$-geodesic map if and only if:

$$
\begin{aligned}
& \frac{\partial^{2} \phi^{A}}{\partial y^{i} \partial y^{\bar{\jmath}}}-{ }^{N} \Gamma_{\bar{\imath} \bar{\jmath}}^{C} \frac{\partial \phi^{A}}{\partial y^{C}}+{ }^{X} \Gamma_{J L}^{A} \frac{\partial \phi^{J}}{\partial y^{i}} \frac{\partial \phi^{L}}{\partial y^{\bar{\jmath}}}=0 \\
& \forall A \in\{1, \overline{1}, \ldots, r, \bar{r}\} \text { and } \forall i, j \in\{1, \ldots, n\} .
\end{aligned}
$$

As we supposed $\phi$ to be holomorphic, Equation (6) is equivalent to:

$$
-{ }^{N} \Gamma_{\bar{i}}^{k} \frac{\partial \phi^{l}}{\partial y^{k}}+{ }^{X} \Gamma_{m \bar{p}}^{l} \frac{\partial \phi^{m}}{\partial y^{i}} \frac{\partial \phi^{\bar{p}}}{\partial y^{\bar{\jmath}}}=0 \quad \forall l \in\{1, \ldots, r\} \text { and } \forall i, j \in\{1, \ldots, n\},
$$

The proof consists of three steps: first we show that ${ }^{N} \Gamma_{\bar{i}}^{k}=0 \quad \forall i, j, k$ such that $k \neq i$ or $k \neq j$, then we establish that ${ }^{X} \Gamma_{m \bar{p}}^{l}=0 \quad \forall l, m, p$, and finally we prove that ${ }^{N} \Gamma_{\bar{i}}^{k}=0 \quad \forall i, j, k$. We work at a point of $N$.

Step 1) Fix the indices $l, i$ and $j$. Equation (7) is:

$$
-{ }^{N} \Gamma_{\bar{\imath}}^{k} \frac{\partial \phi^{l}}{\partial y^{k}}+{ }^{X} \Gamma_{m \bar{p}}^{l} \frac{\partial \phi^{m} \partial \phi^{\bar{p}}}{\partial y^{i} \partial y^{\bar{\jmath}}}=0,
$$

so choosing a holomorphic map $\phi$ such that $\frac{\partial \phi^{A}}{\partial y^{I}}=\delta_{I k_{0}}^{A l}$ with $k_{0} \neq i$ or $k_{0} \neq j$ (possible if $n>1$ ), (7) becomes:

$$
{ }^{N} \Gamma_{\bar{i}}^{k_{0}}=0,
$$

which proves Step 1. We remark that if $n=1$, any Hermitian metric on $N$ is Kähler (cf. [7]) so ${ }^{N} \Gamma_{\bar{i}}^{k}=0 \quad \forall i, j, k$.

Step 2) Consider Equation (7) when $i \neq j$. In this case, whichever value $k$ takes, either $k \neq i$ or $k \neq j$. Because of Step 1) (and the remark for $n=1$ ), with those indices, (7) reads:

$$
{ }^{X} \Gamma_{m \bar{p}}^{l} \frac{\partial \phi^{m} \partial \phi^{\bar{p}}}{\partial y^{i} \partial y^{\bar{\jmath}}}=0 .
$$


Fix $m_{0}, p_{0}$ and choose $\phi$ holomorphic such that $\frac{\partial \phi^{M}}{\partial y^{T}}=\delta_{I i}^{M m_{0}}+\delta_{I j}^{M p_{0}}$. For this map Equation (7) yields

$$
{ }^{x} \Gamma_{m_{0} \overline{p_{0}}}^{l}=0,
$$

and this shows Step 2).

Step 3) Because of Step 2) Equation (7) now reads:

$$
{ }^{N} \Gamma_{\bar{\imath}}^{k} \frac{\partial \phi^{l}}{\partial y^{k}}=0 .
$$

Choosing a holomorphic map such that $\frac{\partial \phi^{M}}{\partial y^{I}}=\delta_{I k_{0}}^{M l_{0}}$ proves that

$$
{ }^{N} \Gamma_{\bar{i} \bar{j}}^{k_{0}}=0 \quad \forall k_{0}, i, j
$$

and in particular that

$$
{ }^{N} \Gamma_{k \bar{k}}^{k}=0 \quad \forall k,
$$

ending Step 3). This proves that if \pm holomorphic maps are $(1,1)$-geodesic then $X^{r}$ and $N^{n}$ must be $(1,2)$-symplectic manifolds and therefore Kähler.

The converse assertion is clear from the definitions of $(1,2)$-symplectic manifolds, (1,1)-geodesic and \pm holomorphic maps.

We now give an example where the notions of $(1,1)$-geodesic and pluriharmonic are distinct.

Example 1. The Hopf manifold (cf. [7, Vol. II]) is defined as $H=\left(C^{n}-\{0\} / \Delta_{\lambda}\right)(n>1)$, where $\Delta_{\lambda}$ is the group generated by the transformation $z \mapsto \lambda z, \quad \lambda \in \mathrm{C}-\{0,1\}$.

$H$ is diffeomorphic to $\mathrm{S}^{1} \times \mathrm{S}^{2 n-1}$, and is a compact Hermitian manifold equipped with the metric

$$
d s^{2}=\frac{1}{\sum_{k=1}^{n} z^{k} z^{k}} \sum_{j=1}^{n} d z^{j} \otimes d z^{\bar{j}} .
$$

Its fundamental Kähler form $\Omega$ is

$$
\Omega=\frac{-i}{\pi \sum_{k=1}^{n} z^{k} z^{k}} \sum_{j=1}^{n} d z^{j} \wedge d z^{\bar{j}} .
$$

The manifold $H$ is not Kähler since its first Betti number $b_{1}(H)$ is equal to $b_{1}\left(\mathrm{~S}^{1} \times \mathrm{S}^{2 n-1}\right)$ which is 1 , whereas the Betti numbers $b_{2 n+1}$ of a Kähler manifold are even.

A simple computation shows that 


$$
\begin{aligned}
& \Gamma_{i \bar{\jmath}}^{k}=\frac{1}{2|z|^{2}}\left(\delta_{i j} z^{k}-\delta_{i k} z^{j}\right) \\
& \Gamma_{i \bar{\jmath}}^{\bar{k}}=\frac{1}{2|z|^{2}}\left(\delta_{i j} z^{\bar{k}}-\delta_{j k} z^{\bar{\imath}}\right)
\end{aligned}
$$

therefore

$$
\begin{aligned}
& \frac{\partial^{2} \phi}{\partial z^{i} \partial z^{\bar{\jmath}}}-\Gamma_{\bar{i}}^{k} \frac{\partial \phi}{\partial z^{k}}-\Gamma_{i \bar{\jmath}}^{\bar{k}} \frac{\partial \phi}{\partial z^{\bar{k}}}= \\
& \frac{\partial^{2} \phi}{\partial z^{i} \partial z^{\bar{\jmath}}}+\frac{1}{2|z|^{2}}\left[z^{j} \frac{\partial \phi}{\partial z^{i}}+z^{\bar{\imath}} \frac{\partial \phi}{\partial z^{\bar{\jmath}}}-\delta_{i j} \sum_{k}\left(z^{\bar{k}} \frac{\partial \phi}{\partial z^{\bar{k}}}+z^{k} \frac{\partial \phi}{\partial z^{k}}\right)\right] .
\end{aligned}
$$

If $\phi$ is a (1,1)-geodesic function on $H$ then

$$
\frac{\partial^{2} \phi}{\partial z^{i} \partial z^{\bar{\jmath}}}-\Gamma_{i \bar{\jmath}}^{k} \frac{\partial \phi}{\partial z^{k}}-\Gamma_{i \bar{\jmath}}^{\bar{k}} \frac{\partial \phi}{\partial z^{\bar{k}}}=\quad 0 \quad \forall i, j=1, \ldots, n .
$$

In the case where $\phi$ is holomorphic Equation $\left({ }^{* *}\right)$, for $i \neq j$, becomes:

$$
z^{j} \frac{\partial \phi}{\partial z^{i}}=0
$$

showing that $\phi$ has to be constant.

We have shown

Proposition 5. Any local \pm holomorphic $(1,1)$-geodesic function on $H$ is constant.

REMARK 3. Since $H$ is Hermitian, any (non-constant) \pm holomorphic function on $H$ is pluriharmonic, therefore local pluriharmonic functions exist.

Finally we compare $(1,1)$-geodesic maps and pluriharmonic maps on Hermitian manifolds and the situation is what the last two propositions suggest:

Proposition 6. Let $N^{n}$ be a Hermitian manifold and $X^{r}$ a Kähler manifold, then the condition "a map from $N^{n}$ to $X^{r}$, is a (local) $(1,1)$-geodesic map if and only if it is pluriharmonic" is equivalent to $N$ being Kähler.

Proof. The definitions of $(1,1)$-geodesic map and pluriharmonic map for $\phi: N^{n} \rightarrow X^{r}$ coincide when:

ProOF.

$$
{ }^{N} \Gamma_{\bar{\imath}}^{C} \frac{\partial \phi^{I}}{\partial y^{C}}=0 \quad \forall I \in\{1, \overline{1}, \ldots, r, \bar{r}\} \quad \forall i, j \in\{1, \ldots, n\} .
$$


As $X^{r}$ is Kähler, \pm holomorphic maps from $N^{n}$ to $X^{r}$ are pluriharmonic (Proposition 3). We choose $\phi$ holomorphic and such that

$$
\frac{\partial \phi^{A}}{\partial y^{C}}=\delta_{C k_{0}}^{A \alpha}
$$

then Equation (9) reads:

$$
{ }^{N} \Gamma_{\bar{i} \bar{j}}^{k_{0}}=0 \quad \forall i, j \in\{1, \ldots, n\} .
$$

Since this holds for any $k_{0}=1, \ldots, n, N$ is $(1,2)$-symplectic and so Kähler since integrable.

Remark 4. In Propositions 3, 4 and 6 the need for the integrability of the complex structures on $N^{n}$ and $X^{r}$ is in the use of $n$ linearly independent holomorphic functions as "test functions", the existence of these being equivalent to the integrability of the structures.

Proposition 7. [9] Let $(N, J, g)$ be a Hermitian manifold and $(X, h)$ an almost Hermitian manifold. Then $(N, J, g)$ is cosymplectic if and only if any pluriharmonic map $\phi:(N, J, g) \rightarrow(X, h)$ is harmonic.

Definition 5. We shall call $\psi$ a pluriharmonic morphism if it pulls back (local) pluriharmonic functions to (local) pluriharmonic functions, i.e. for all functions $\phi$ satisfying $\nabla^{(0,1)} d^{\prime} \phi=0$ we have

$$
\nabla^{(0,1)} d^{\prime}(\phi \circ \psi)=0 .
$$

REMARK 5. Since a complex-valued function is pluriharmonic if and only if its real and imaginary parts are real-valued pluriharmonic functions, we can choose to consider, in the above definition, real-valued or complex-valued functions.

Lemma 1. Let $N$ be a complex manifold. Given $p \in N$ and any constants $\left(C_{A}\right)_{A=1, \overline{1}, \ldots, n, \bar{n}}$ and $\left(C_{A B}\right)_{A, B=1, \overline{1}, \ldots, n, \bar{n}, \text { type } A=\text { type } B}$ such that $C_{A B}=C_{B A}$, there exists a (complex-valued) pluriharmonic function, $h$, defined on a neighbourhood of p satisfying $\frac{\partial h}{\partial y^{4}}(p)=C_{A}$ and $\frac{\partial^{2} h}{\partial y^{A} \partial y^{B}}(p)=C_{A B}$.

Proof. We choose complex local coordinates $\left(y^{1}, y^{\overline{1}}, \ldots, y^{n}, y^{\bar{n}}\right)$ in a neighbourhood of the point $p \in N$.

We shall look for pluriharmonic functions; in this case, without loss of generality we can take $N=\mathrm{C}^{n}$ and $X=\mathrm{C}$. This is then easy, for example:

$$
h(z)=\sum_{i=1}^{n} C_{i} y^{i}+\frac{1}{2} \sum_{i, j=1}^{n} C_{i j} y^{i} y^{j}+\sum_{\bar{i}=\overline{1}}^{\bar{n}} C_{\bar{l}} y^{\bar{i}}+\frac{1}{2} \sum_{\bar{\imath}, \bar{j}=\overline{1}}^{\bar{n}} C_{\bar{\imath}} y^{\bar{y}} y^{\bar{\jmath}},
$$


where the $C_{i}, C_{\bar{\imath}}$ and $C_{i, j}, C_{\bar{\imath}}$ are complex constants, is pluriharmonic, since it is the sum of a holomorphic function and an anti holomorphic function, and has the prescribed first and second derivatives.

Proposition 8. Let $\psi$ be a smooth map from a complex manifold $M$ to a complex manifold $N$. Then $\psi$ is a pluriharmonic morphism if and only if it is a \pm holomorphic map.

Proof. Let $\psi: M \rightarrow N$ be a pluriharmonic morphism, then it must satisfy

$$
\nabla^{(0,1)} d^{\prime}(\phi \circ \psi)(\bar{Z}, W)=0
$$

for all $Z, W \in T^{(1,0)} M$ and all local pluriharmonic functions $\phi: N \rightarrow C$.

We first compute the chain rule for the operator $\nabla^{(0,1)} d^{\prime}$.

Recall that:

$$
d^{\prime}(\phi \circ \psi)=\left.d(\phi \circ \psi)\right|_{T^{(1,0)} M}=\left.d \phi \circ d \psi\right|_{T^{(1,0)} M}=d \phi \circ d^{\prime} \psi .
$$

Hence

$$
\begin{aligned}
& \nabla^{(0,1)} d^{\prime}(\phi \circ \psi)(\bar{Z}, W)= \\
& { }^{\phi \circ \psi} \nabla_{\bar{Z}}\left(d^{\prime}(\phi \circ \psi)(W)\right)-d^{\prime}(\phi \circ \psi)\left(\bar{\partial}_{\bar{Z}} W\right)= \\
& { }^{\phi \circ \psi} \nabla_{\bar{Z}}\left(d \phi \circ d^{\prime} \psi(W)\right)-d \phi \circ d^{\prime} \psi\left(\bar{\partial}_{\bar{Z}} W\right)= \\
& \left({ }^{\phi} \nabla_{d^{\prime \prime} \psi(\bar{Z})} d \phi\right)\left(d^{\prime} \psi(W)\right)+d \phi\left({ }^{\psi} \nabla_{\bar{Z}}(d \psi(W))-d \phi \circ d^{\prime} \psi\left(\bar{\partial}_{\bar{Z}} W\right)=\right. \\
& (\nabla d \phi)\left(d^{\prime \prime} \psi(\bar{Z}), d^{\prime} \psi(W)\right)+d \phi\left[{ }^{\psi} \nabla_{\bar{Z}}(d \psi(W))-d^{\prime} \psi\left(\bar{\partial}_{\bar{Z}} W\right)\right]= \\
& {\left[\nabla d \phi\left(d^{\prime \prime} \psi, d^{\prime} \psi\right)+d \phi\left(\nabla^{(0,1)} d^{\prime} \psi\right)\right](\bar{Z}, W) .}
\end{aligned}
$$

If we choose $Z, W$ to be the vectors $\frac{\partial}{\partial z^{i}}, \frac{\partial}{\partial z^{\prime}}$ of the canonical frame of $T^{(1,0)} M$ :

$$
\begin{aligned}
& \nabla^{(0,1)} d^{\prime}(\phi \circ \psi)\left(\frac{\partial}{\partial z^{\bar{\jmath}}}, \frac{\partial}{\partial z^{i}}\right)= \\
& \frac{\partial \phi}{\partial y^{C}}\left[\frac{\partial^{2} \psi^{C}}{\partial z^{i} \partial z^{\bar{\jmath}}}+{ }^{N} \Gamma_{J L}^{C} \frac{\partial \psi^{J}}{\partial z^{i}} \frac{\partial \psi^{L}}{\partial z^{\bar{j}}}\right]+ \\
& \sum_{A, B=1, \overline{1}}^{n, \bar{n}} \frac{\partial \psi^{A}}{\partial z^{i}} \frac{\partial \psi^{B}}{\partial z^{\bar{\jmath}}}\left[\frac{\partial^{2} \phi}{\partial y^{A} \partial y^{B}}-{ }^{N} \Gamma_{A B}^{C} \frac{\partial \phi}{\partial y^{C}}\right]= \\
& \frac{\partial \phi}{\partial y^{C}} \frac{\partial^{2} \psi^{C}}{\partial z^{i} \partial z^{\bar{\jmath}}}+\frac{\partial \psi^{A}}{\partial z^{i}} \frac{\partial \psi^{B}}{\partial z^{\bar{\jmath}}} \frac{\partial^{2} \phi}{\partial y^{A} \partial y^{B}}
\end{aligned}
$$

The "if" part of the proposition follows from (10), since if $\psi$ is holomorphic then 


$$
\begin{array}{cl}
\frac{\partial \psi^{\alpha}}{\partial z^{i}} \frac{\partial \psi^{\beta}}{\partial z^{\bar{\jmath}}}=0 & \forall \alpha, \beta=1, \ldots, n \\
\frac{\partial^{2} \psi^{C}}{\partial z^{i} \partial z^{\bar{\jmath}}}=0 & \forall C=1, \overline{1}, \ldots, n, \bar{n} .
\end{array}
$$

Conversely, by Lemma 1 we can choose various different values for $\frac{\partial \phi}{\partial y^{C}}$ and $\frac{\partial^{2} \phi}{\partial y^{B} \partial y^{A}}$ ( $A$ and $B$ of the same type) at a given point:

If we take $\frac{\partial^{2} \phi}{\partial y^{B} \partial y^{A}}=0$ for all $A, B$ and $\frac{\partial \phi}{\partial y^{C}}=0$ for all $C$ except for $l_{0}$ (we will suppose $\frac{\partial \phi}{\partial y^{\prime} 0}=1$ ) then Equation (10) becomes:

$$
\frac{\partial^{2} \psi^{l_{0}}}{\partial z^{i} \partial z^{\bar{\jmath}}}=0
$$

for all $l_{0} \in\{1, \overline{1}, \ldots, n, \bar{n}\}$. If we take $\phi$ such that $\frac{\partial \phi}{\partial y^{C}}=0$ for all $C \in\{1, \overline{1}, \ldots, n, \bar{n}\}$ and $\frac{\partial^{2} \phi}{\partial y^{B} \partial y^{A}}=0$ for all $A, B$ but for $\alpha_{0}, \beta_{0} \stackrel{\partial y}{\in}\{1, \ldots, n\}$ (we will suppose that $\frac{\partial^{2} \phi}{\partial y^{\beta_{0}} \partial y^{\alpha_{0}}}=1$ ), Equation (10) becomes:

$$
\frac{\partial \psi^{\beta_{0}} \partial \psi^{\alpha_{0}}}{\partial z^{\bar{\jmath}} \partial z^{i}}=0
$$

which we obtain for all $\alpha_{0}, \beta_{0}=1, \ldots, n, i, j=1, \ldots, m$.

Either (i) for all $\alpha=1, \ldots, n$, and all $i$ :

$$
\frac{\partial \psi^{\alpha}}{\partial z^{i}}=0
$$

or (ii) there exist $\alpha_{0}$ and $i_{0}$ such that:

$$
\frac{\partial \psi^{\alpha_{0}}}{\partial z^{i_{0}}} \neq 0
$$

Then Equation (12) shows that:

$$
\frac{\partial \psi^{\alpha}}{\partial z^{\bar{\imath}}}=0
$$

for all $\alpha=1, \ldots, n$ and all $i$.

Therefore at each point $p \in M, \psi$ is either antiholomorphic or holomorphic.

We shall use an argument of Siu [11] to prove that $\psi$ is either holomorphic or antiholomorphic on the whole of $M$.

Let

$$
\begin{aligned}
& U=\{p \in M \quad \mid \quad \partial \psi(p) \equiv 0\} \\
& V=\{p \in M \quad \mid \quad \bar{\partial} \psi(p) \equiv 0\} .
\end{aligned}
$$

As 


$$
U \cup V=M,
$$

either $\stackrel{\circ}{U} \neq \emptyset$ or $\stackrel{\circ}{V} \neq \emptyset$. Suppose that $\stackrel{\circ}{V} \neq \emptyset$ and let $\widetilde{V}$ be the largest connected open subset of $M$ included in $V$.

If $M=\widetilde{V}$ then $\psi$ is holomorphic. Assume that $M \neq \widetilde{V}$. Let $p$ be a boundary point of $V$. Let $W$ be an open connected neighbourhood of $p$ in $M$ such that:

1. there exists a holomorphic coordinate system $\left(z^{i}\right)$ on some open neighbourhood of $\bar{W}$,

2. there exists a holomorphic coordinate system $\left(w^{\alpha}\right)$ on some open neighbourhood of $\overline{\psi(W)}$.

Since $\psi$ satisfies Equation (11):

$$
\begin{aligned}
& \frac{\partial^{2} \psi^{\alpha}}{\partial z^{i} \partial z^{j}}=0 \\
& \forall \alpha \in\{1, \ldots, r\} \text { and } \forall i, j \in\{1, \ldots, n\} .
\end{aligned}
$$

Applying the operator $\partial_{\bar{k}}$ to the trace of (13) yields:

$$
\left|{ }^{M} \Delta\left(\frac{\partial \psi^{\alpha}}{\partial z^{\bar{k}}}\right)\right| \leq C\left(\sum_{i, j}\left|\frac{\partial^{2} \psi^{\alpha}}{\partial z^{i} \partial z^{\bar{\jmath}}}\right|+\sum_{j, L}\left|\frac{\partial \psi^{L}}{\partial z^{\bar{\jmath}}}\right|+\sum_{j, J}\left|\frac{\partial^{2} \psi^{J}}{\partial z^{\bar{k}} \partial z^{\bar{\jmath}}}\right|\right),
$$

where ${ }^{M} \Delta=-2 \sum_{i, j} g^{i \bar{j}} \frac{\partial^{2}}{\partial z^{i} \partial z^{\bar{j}}}$ and $C$ is a positive constant.

Set

$$
\frac{\partial \psi^{\alpha}}{\partial z^{\bar{k}}}=u_{\bar{k}}^{\alpha}+i v_{\bar{k}}^{\alpha}
$$

Then Equation (13) means that:

$$
\left|{ }^{M} \Delta u_{\bar{k}}^{\alpha}\right|^{2}+\left|{ }^{M} \Delta v_{\bar{k}}^{\alpha}\right|^{2} \leq C^{\prime} \sum_{\beta, j}\left\{\left|\operatorname{grad}\left(u_{\bar{\jmath}}^{\beta}\right)\right|^{2}+\left|\operatorname{grad}\left(v_{\bar{\jmath}}^{\beta}\right)\right|^{2}+\left|u_{\bar{\jmath}}^{\beta}\right|^{2}+\left|v_{\bar{\jmath}}^{\beta}\right|^{2}\right\}
$$

on $W$. Applying Aronszajn's unique continuation Theorem [1] shows that since $u_{\bar{\jmath}}^{\beta}=v_{\bar{\jmath}}^{\beta}=0$ on $W \cap \widetilde{V}$ then $u_{\bar{\jmath}}^{\beta}=v_{\bar{\jmath}}^{\beta}=0$ on $\widetilde{W}$. But $\widetilde{V}$ was supposed to be maximal therefore $\psi$ is holomorphic.

The anti-holomorphic case is similar.

Proposition 9. A pluriharmonic morphism from a Hermitian manifold to a Kähler manifold pulls back (local) pluriharmonic maps to (local) pluriharmonic maps.

Proof. Let $\psi: M \rightarrow N$ be a pluriharmonic morphism (i.e. a \pm holomorphic map) and $\phi: N \rightarrow P$ a pluriharmonic map. Since $N$ is Kähler, $\psi$ is a pluriharmonic map. 
The map $\phi \circ \psi$ is pluriharmonic if

$$
\nabla^{(0,1)} d^{\prime}(\phi \circ \psi)(\bar{Z}, W)=0 \quad \forall Z, W \in T^{(1,0)} M .
$$

But

$$
\begin{aligned}
& \nabla^{(0,1)} d^{\prime}(\phi \circ \psi)(\bar{Z}, W)= \\
& d \phi \circ \nabla^{(0,1)} d^{\prime} \psi(\bar{Z}, W)+\nabla d \phi(d \psi(\bar{Z}), d \psi(W))= \\
& \quad \text { as shown in Proposition } 8 \\
& \nabla d \phi(d \psi(\bar{Z}), d \psi(W))= \\
& \nabla_{d \psi(\bar{Z})} d \phi(d \psi(W))-d \phi\left(\nabla_{d \psi(\bar{Z})} d \psi(W)\right) .
\end{aligned}
$$

Suppose that $\psi$ is holomorphic then $d \psi(W) \in T^{(1,0)} N$, therefore

$$
d \phi(d \psi(W))=d^{\prime} \phi(d \psi(W))
$$

and, as the bundle $T^{(1,0)} N \rightarrow N$ is holomorphic,

$$
\nabla_{d \psi(\bar{Z})} d \psi(W)=\bar{\partial}_{d \psi(\bar{Z})} d \psi(W) .
$$

Therefore the right-hand side of (15) can be written

$$
\nabla^{(0,1)} d^{\prime} \phi(d \psi(\bar{Z}), d \psi(W))
$$

which vanishes since $\phi$ is pluriharmonic.

\section{REFERENCES}

1. N. Aronszajn, A unique continuation theorem for solutions of elliptic partial differential equations or inequalities of second order, J. Math. Pures Appl. 9 (1957), 235-249.

2. J. Eells and L. Lemaire, A report on harmonic maps, Bull. London Math. Soc. 10 (1978), $1-68$.

3. J. Eells and L. Lemaire, Another report on harmonic maps, Bull. London Math. Soc. 20 (1988),385-524.

4. B. Fuglede, Harmonic morphisms between Riemannian manifolds, Ann. Inst. Fourier (Grenoble) 28 (1978), 107-144.

5. A. Gray and L. M. Hervella, The sixteen classes of almost Hermitian manifolds and their linear invariants, Ann. Mat. Pura Appl. 123 (1980), 35-58.

6. T. Ishihara, A mapping of Riemannian manifolds which preserves harmonic functions, $\mathbf{J}$. Math. Kyoto Univ. 19, (1979), 215-229.

7. S. Kobayashi and K. Nomizu, Foundations of Differential Geometry, I and II, volume 15 of Interscience Tracts in Pure and Applied Mathematics, Interscience, 1963, 1969.

8. A. Lichnerowicz, Applications harmoniques et variétés kählériennes, Sympos. Math. (1970), 341-402.

9. Y. Ohnita and G. Valli, Pluriharmonic maps into compact Lie groups and factorization into unitons, Proc. London Math. Soc. 61 (1990), 546-570. 
10. S.M. Salamon, Harmonic and holomorphic maps, In E, Vesentini, editor, Geometry Seminar "Luigi Bianchi”' I-1984, Lecture Notes in Math. 1164 (1985), 161-224.

11. Y.T. Siu, The complex-analyticity of harmonic maps and the strong rigidity of compact Kähler manifolds, Ann. of Math. 112 (1980), 73-111.

12. S. Udagawa, Pluriharmonic maps and minimal immersions of Kähler manifolds, J. London Math. Soc. 37 (1988), 375-384.

13. S. Udagawa, Classification of pluriharmonic maps from compact complex manifolds with positive first Chern class into complex Grassmann manifolds, Tôhoku Math. J. 46 (1994), $367-391$.

UNIVERSITÉ DE BRETAGNE OCCIDENTALE

UFR SCIENCES ET TECHNIQUES

DEPARTEMENT DE MATHEMATIQUES

6, AVENUE VICTOR LE GORGEU

BP 809

29285 BREST CEDEX

FRANCE

Email: loubeau@univ-brest.fr 